\title{
Towards understanding learning challenges involving sign convention in introductory level kinematics
}

\author{
Moa Eriksson ${ }^{1,2}$, Cedric Linder ${ }^{2}$, Urban Eriksson ${ }^{1}$ \\ ${ }^{1}$ Department of Physics, Lund University, Lund, Sweden, 22100 \\ ${ }^{2}$ Department of Physics and Astronomy, Uppsala University, Uppsala, Sweden, 75120
}

\begin{abstract}
Coming to appropriately appreciate the meaning of algebraic signs is an important aspect in introductory kinematics. However, in this educational context, the "disciplinary relevant aspects" of algebraic signs across vector and scalar representations are extremely difficult to discern. Our study explores the "relevance structure" that one-dimensional kinematics problems evoked for introductory level university physics students across two very different educational systems which have, in PER terms, progressive teaching environments: Sweden $(n=60)$ and South Africa $(n=24)$. The outcomes of two previous PER studies are used to provide the analytic basis for formulating categories of relevance structure. Aspects of a contemporary PER-developed social semiotics perspective (referred to here in terms of communication practices) are used to discuss implications for teaching in the given educational context of introductory kinematics.
\end{abstract}

\section{INTRODUCTION}

Research aimed at understanding learning challenges involving algebraic signs in introductory level kinematics has been rare. However, algebraic signs have been identified as presenting a significant learning challenge for physics students [1-4]. The aim of the research reported on here is to contribute to addressing this deficit by drawing on the idea that a core part of the process of learning involves students learning to "read" the situated physics-ways of representing disciplinary relevant aspects in order to understand and use them appropriately [5-10]. From this perspective, such "reading" is thought of as being about recognizing a situation that requires being experienced in a particular disciplinary way. In other words, there is a disciplinary awareness - a discernment - that is called for that is directly related to the context, which includes the way the parts of this awareness are seen to relate to one another [5,9] (i.e. how it gets read in relation to how it gets used). The context is central here because what emerges in a person's "focal awareness" for a given context is a function of the experience of that context (both past and present) and how that experience has been made sense of. The way a person "reads" a situation can be analytically referred to in the broader "student learning" literature as a person's relevance structure. This is because relevance structure is "the person's experience of what the situation calls for, what it demands. It is a sense of aim, of direction, in relation to which different aspects of the situation appear more or less relevant" [11] - what gets focused on by them in a given context. Thus, for the purposes of our study the concept of relevance structure presents an opportunity to further explore learning challenges involving algebraic signs by using a study situated in introductory level kinematics problem-solving regardless of where the education takes place.

${ }^{1}$ The collective-level points to "identifying the very [actual] ways in which something may be experienced" [11]

\section{A. Research question}

Our study posed the following research question: What are the individual-level categories of variation of experiencing relevance structure with regard to algebraic signs $(+$ and -$)$ in introductory kinematics problem-solving in university physics education?

\section{METHOD}

Our method built on the analysis of a set of collectivelevel ${ }^{1}$ categories obtained by Eriksson [12], which drew on the work by Govender [2,3], in the area of introductory kinematics. Our point of departure from these studies was bringing the focus of interpretation to the individual level for the purpose of iteratively [13] constructing categories of relevance structure [14].

The participating students were drawn from two introductory physics classes, one at a Swedish university and the other in a South African. These classes were chosen on the basis that the educational environments in both settings were, from a PER perspective, progressive (in terms of the teacher being familiar with the educational benefits associated with knowledge of PER) and that the students in these classes came from different socio-economic backgrounds. In total, 84 students participated with appropriate ethical consent. Both groups of students had completed coursework that involved using algebraic signs for vector calculations in kinematics.

The data set was made up of a combination of students' individual written questionnaire answers and verbatim transcriptions of follow-up interview discussions.

The analysis proceeded as follows: first, a questionnaire was given to the students which contained two onedimensional straight-line scenarios (see Fig. 1) where

and since this was the principal aim of our study, no individual classification was done. 
students were asked to explain the meaning of any algebraic signs that they used in their expressions of displacement, distance, speed, velocity, and acceleration for a constant velocity situation; and, velocity and acceleration for a changing velocity situation. From this set of answers a "purposeful sample" [15] of students was chosen for semistructured follow-up interviews about the way(s) they had answered these two questions - a stimulated recall research procedure [16]. This combined data set was then used to construct categories of relevance structure. In line with the epistemological stance that grounded our study [11], the procedure for this followed a naturalistic qualitative analytic method [13] in that it involved a constant comparative approach made up of emerging coding in relation to sorting into category groups. This ended when redundancy was reached. The Swedish and South African data were coded separately and then merged together for the final analysis. The entire coding process was independently cross-checked by PER colleagues.

\section{RESULTS}

Our methodology yielded four different categories of relevance structure (see Table I) that the participating introductory level physics students evoked when reading and using signs $(+$ and -$)$ in one-dimensional kinematics problems. The same variation of relevance structure was observed for both the Swedish and the South African data sets, i.e. they were independent of educational context.

We propose that the categories have an inherent hierarchy that spans from the least advanced way of reading and using signs (Category A) to the most advanced way (Category D).

TABLE I. Categories of relevance structure for algebraic signs in introductory level kinematics.

\begin{tabular}{|c|c|c|}
\hline $\begin{array}{l}\text { Category of } \\
\text { relevance } \\
\text { structure: }\end{array}$ & $\begin{array}{l}\text { "Reading" - } \\
\text { Variation } \\
\text { described in: }\end{array}$ & $\begin{array}{l}\text { "Use" - } \\
\text { Focus of intention } \\
\text { (relevance structure } \\
\text { perceived): }\end{array}$ \\
\hline A & $\begin{array}{l}\text { No specific } \\
\text { assignment of } \\
\text { signs }\end{array}$ & $\begin{array}{l}\text { - Nothing necessarily } \\
\text { specific in } \\
\text { kinematics terms }\end{array}$ \\
\hline B & $\begin{array}{l}+ \text { and }- \\
\text { assignment } \\
\text { (single purpose) }\end{array}$ & $\begin{array}{l}\text { - Representing } \\
\text { changing } \\
\text { magnitude }\end{array}$ \\
\hline $\mathrm{C}$ & $\begin{array}{l}+ \text { and - } \\
\text { assignment } \\
\text { (dual purpose) }\end{array}$ & $\begin{array}{l}\text { - Representing } \\
\text { magnitude in the } \\
\text { case of acceleration } \\
\text { - Representing } \\
\text { direction in the } \\
\text { case of velocity }\end{array}$ \\
\hline $\mathrm{D}$ & $\begin{array}{l}+ \text { and }- \\
\text { assignment } \\
\text { (dual purpose) }\end{array}$ & $\begin{array}{l}\text { - Representing } \\
\text { direction by } \\
\text { convention } \\
\text { - Representing } \\
\text { direction by choice }\end{array}$ \\
\hline
\end{tabular}

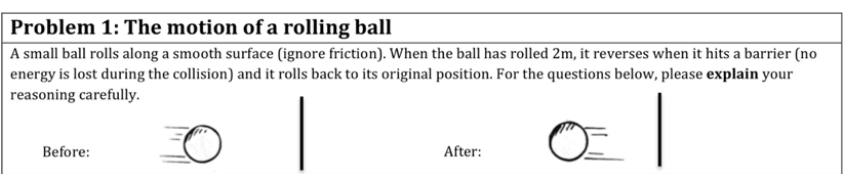

FIG 1. The first problem given on the questionnaire. The problem context is the following: "A small ball rolls along a smooth surface (ignore friction). When the ball has rolled $2 \mathrm{~m}$, it reverses when it hits a barrier (no energy is lost during the collision) and it rolls back to its original position."

In the summary description of the results that follow, we present selected excerpts from the data that make up the different relevance structure categories.

Note: Q and I refer to questionnaire and interview sources respectively, while S refers to student's answers from both. Each "box" represents a stand-alone example.

For the purposes of some of the examples used in this paper, the Swedish has been translated into English.

\section{A. Algebraic signs do not necessarily have specific relevance in kinematics}

Students that experience this relevance structure do not consider algebraic signs to be needed specifically in kinematics. This is because directional signs can be replaced by directly referring to the given direction linguistically. For example:

Q: Explain the speed and velocity of the ball before and after it turns. Explain the meaning of any algebraic signs (+ and -$)$ that you use.

S: [...] I think that + and - seems a bit unnecessary. Why don't [we] just say a motion to the right or left?

I: Would [the velocity] have any signs in this case?
S: No, not when we have decided forward as left. I don't
really think in terms of plus and minus, but I think in terms
of right and left.

\section{B. When an algebraic sign is assigned to a kinematic unit it is seen as being relevant for representing a changing magnitude}

Students that experience this relevance structure appear to be connecting their way of reading algebraic signs in everyday life in relation to getting bigger or smaller. For example:

I: What do you think that the signs for velocity show? $\mathrm{S}$ : Plus to me means that it is going faster, that the velocity increases. And minus should then be the opposite, that the velocity simply decreases.

S: I experience plus as something that is getting bigger and minus as something that is getting smaller.

This category of relevance structure illustrates how students can get to experience signs as having a single purpose of indicating changing magnitude, often connecting 
the sign for acceleration to an increase or decrease of velocity and vice versa.

I: What does the signs for acceleration mean to you?
S: Increase or decrease of velocity.

I: So when [the car] goes from some velocity to no velocity [what does it mean to you]?

$\mathrm{S}$ : Then it is deceleration, I'm thinking negative acceleration.

\section{The assignment of algebraic signs in kinematics has dual relevance: for representing changing magnitudes in the case of acceleration, and for representing direction in the case of velocity}

Students that experience this relevance structure see algebraic signs as having a dual purpose in kinematics. In the case of velocity, they indicate direction, and in the case of acceleration they indicate magnitudes.

S: [I]n velocity the signs only specify the direction of
motion, however in acceleration it means speeding [up] or
slowing [down].

$\mathrm{S}$ : [W] hen it comes to velocity + and - only show direction. When it comes to acceleration they only show the acceleration's increase or decrease and don't take direction into consideration. Why it turned out this way I don't know!

\section{The assignment of algebraic signs in kinematics has dual relevance: for representing direction by convention, and for representing direction by choice}

This category of relevance structure illustrates how students can get to experience signs as being functional for indicating how direction gets to be assigned; by convention or by choice. Direction by convention is often stated explicitly, as illustrated in the following examples:

Q: Is the direction important to be able to decide the motion of the ball? Explain.

$\mathrm{S}:[\ldots]$ The motion is positive, if the direction of the ball is to the right [as] in this case.

Q: Is there any difference to the motion of the ball before and after the turn? Explain the meaning of any algebraic signs (+ or -) that you used, if any.

$\mathrm{S}$ : The motion is negative (-) after [the turn]. Because its motion is in the opposite direction.

Similarly, representing direction by choice is illustrated in the following transcript excerpts:

I: And what do the signs mean to you in physics?
S: It is the direction, partly. Or the direction in relation to
how you decide on it.

$\mathrm{S}$ : It is the direction, partly. Or the direction in relation to decide on it.
Q: Is there any difference to the motion of the ball before and after the turn? Explain the meaning of any algebraic signs (+ or - ) that you used, if any.

S: No difference except that directions are opposite. If we choose the initial direction as positive (+) then the other direction after the ball hit the barrier would be negative $(-)$.

\section{DISCUSSION}

Students' relevance structure - how they "read" algebraic signs in kinematics - was explored. The results indicate that the students had largely made sense of how they have experienced algebraic signs being used in kinematics formulations without direct reference to a coordinate system. Furthermore, the idea of calculation-invariance associated with the free choice of a coordinate system was seldom found in the data set. From a social semiotics perspective [17], we propose that this is partially a consequence of a lack of appreciation for the importance that communication practices play in the teaching and learning of physics [e.g. see Ref. 8], such as, in our case, when introducing students to one-dimensional kinematic problem-solving. Discussion with nine introductory physics lecturers from both countries together with the inspection of five popular introductory level textbooks lead us to anecdotally propose the following: that the communication practices used to exemplify problem solving in introductory kinematics have the distinct potential to lead to "fuzzy" readings of algebraic signs and the understanding of their usage in one-dimensional kinematics. Thus, such communication practices arguably contribute to the personal constitution of the range of relevance structure categories presented in this article. Examples of the kind of communication practices that may lead to "fuzziness" that we encountered are:

1. one-dimensional kinematics being dealt with before doing a generalized introduction to vectors while implicitly drawing on fundamentals that arise out of the mathematical application of a coordinate system;

2. the components are not always portrayed as scalars both in words and pictorially. Here, the signs emerge from a parallel or anti-parallel alignment of the so-called "component vector" with the given coordinate axis;

3. the use of unit vector notation as the route to obtaining one-dimensional scalar equations with their correct signs where moving from the unit vector to scalar representation is taken to be unproblematic; and,

4. in stark contrast signs were simply assigned as a convention linked to direction.

In all four of these illustrative communication practices, pictorial representations get used alongside mathematical and linguistic representations, and it appears as if a basic understanding of vector algebra is taken to be self-evident.

There have been several recent PER contributions that have started to model links between gaining access to what is educationally critical - disciplinary relevant aspects [9] - 
for a given object of learning [11] and the representations that get used to share this knowledge (for a summary see Ref. [17]). There has been work that advocates students be explicitly taught to work competently with a set of representational forms and there have been proposals that there is a critical combination of representations (i.e. semiotic resources [17,18] such as graphs, diagrams, sketches, figures, mathematics, specialist language, etc.) that are needed to give an encompassing access to the disciplinary relevant aspects of a given object of learning. From such a social semiotic standpoint, even when an explicit effort is made to prevent the "fuzziness" referred to earlier, other challenging communication practices can emerge, such as drawing a single component coordinate system using a straight line with an arrow head added as some texts do (some texts add a zero to indicate some kind of one-dimensional origin). However, should the teaching practice follow a communicative format [19] to bring out awareness of the disciplinary relevant aspects and to work through the relevance structure categories reported here, then we believe that the learning possibility could be optimized by design. We propose that such design be built on the idea that: in order to learn to experience something in a new and meaningful way, a person needs to become aware of the critical aspects that the new way of experiencing is built on. Such awareness can only become possible through particular communication practices.

In other words, we propose that for such a crafting of practice, teachers need to give more consideration to how the form and content of the representations that get used to make up their communication practices affect the possibility for students to better discern what is important - the disciplinary relevant aspects.

\section{ACKNOWLEDGEMENTS}

We thank Nadaraj Govender for his useful discussions and help with the South African data collection. We also thank Anne Linder and Trevor Volkwyn for their critical reading of drafts of this article. We acknowledge funding support from the Swedish Research Council, 2016-04113.
[1] G. Rebmann and L. Viennot, Am. J. Phys. 62, 723 (1994).

[2] N. Govender, A Phenomenographic Study of Physics Students' Experiences of Sign Conventions in Mechanics (Doctoral thesis, University of Western Cape, Cape Town, South Africa), 1999.

[3] N. Govender, African J. Res. Math. Sci. Technol. Educ. 11, 61 (2007).

[4] S. Brahmia, Phys. Educ. Res. Conf. Proc. (2017)

[5] A. van Heuvelen and X. Zou, Am. J. Phys, 69, 184 (2001).

[6] L. C. McDermott, in Towar. a Sci. Pract. Sci. Educ., edited by M. Gardner, J. G. Greeno, F. Reif, A. H. Schoenfeld, A. A. diSessa, and E. Stage (Lawrence Erlbaum Associates, Hillsdale, 1991), pp. 3-30.

[7] D. E. Meltzer, Am. J. Phys. 73, 463 (2005).

[8] A. Linder, J. Airey, N. Mayaba, and P. Webb, African J. Res. Math. Sci. Technol. Educ. 18, 242 (2014).

[9] T. Fredlund, Using a Social Semiotic Perspective to Inform the Teaching and Learning of Physics (Doctoral thesis, Uppsala University, Sweden), 2015.

[10] U. Eriksson, Reading the Sky: From Starspots to Spotting Stars (Doctoral thesis, Uppsala University, Sweden), 2014.

[11] F. Marton and S. Booth, Learning and Awareness (Lawrence Erlbaum, Mahwah, NJ, 1997).

[12] M. Eriksson, Introductory Physics Students' Conceptions of Algebraic Signs Used in Kinematics Problem Solving (Bachelor's thesis, Uppsala University, Sweden), 2014.

[13] E. G. Guba and Y. S. Lincoln, Naturalistic Inquiry (SAGE, Beverly Hills, CA, 1985).

[14] At a certain level, a methodological relationship can be seen between the PER-established framing-resources perspective [e.g. 14a-c] and the relevance structure construct that we used for our analysis. It was our principle analytic focus - the contextual discernment and the way the parts of this awareness were then seen to relate to one another - that led to our choice of relevance structure. Its strength as an analytic tool here can be seen when consideration is given to its epistemic grounding which is an anatomy of awareness. In contrast, the epistemic grounding of the PER framingresources perspective is discourse analysis (one of the principal sources is [14d]), where the central focus is not necessarily on disciplinary relevant aspects [e.g. $14 \mathrm{e}]$.

[14a]D. Hammer, A. Elby, R. E. Scherr, and E. F. Redish, in Transf. Learn. Res. Perspect., edited by J. Mestre (Information Age Publishing, Greenwich, CT, 2005), pp. 89-119.

[14b]E. F. Redish, Proc. Enrico Fermi Summer Sch. Phys. 1 (2003).

[14c] D. Hammer, Am. J. Phys, 68, S52 (2000).

[14d]D. Tannen, Framing in Discourse (Oxford University Press, New York, 1993).

[14e] P. W. Irving, M. S. Martinuk, and E. C. Sayre, Phys. Rev. Spec. Top. - Phys. Educ. Res. 9, 1 (2013).

[15] M. Q. Patton, Qualitative Evaluation and Research Methods (SAGE, Beverly Hills, CA, 1990).

[16] J. Lyle, Br. Educ. Res. J. 29, 861 (2003).

[17] J. Airey and C. Linder, in Mult. Represent. Phys. Educ., edited by D. F. Treagust, R. Duit, and H. E. Fischer (Springer, 2017), pp. 95-122.

[18] T. van Leeuwen, Introducing Social Semiotics (Routledge, London, 2005).

[19] For example, as described by S. Allie and A. Buffler, Am. J. Phys. 66, 613 (1998). 